OPEN ACCESS

Edited by:

Jianming Li,

Soochow University, China

Reviewed by:

Manidhar Reddy Lekkala, University of Rochester, United States Aaron Wild,

Southeast Radiation Oncology Group, United States

*Correspondence:

Yuejuan Cheng chengyuejuanpumch@163.com Chunmei Bai

baichunmei1964@163.com

${ }^{\dagger}$ These authors have contributed equally to this work

Specialty section:

This article was submitted to Gastrointestinal Cancers, a section of the journal

Frontiers in Oncology

Received: 29 January 2021 Accepted: 06 September 2021 Published: 22 September 2021

Citation:

Xing J, Yang B, Hou X, Jia N, Gong X,

Li X, Zhou N, Cheng Y and Bai C (2021) Prognostic Factors and Effect of Adjuvant Chemoradiation Following

Chemotherapy in Resected Pancreatic Cancer Patients With Lymph Node Metastasis or R1 Resection. Front. Oncol. 11:660215. doi: 10.3389/fonc.2021.660215

\section{Prognostic Factors and Effect of Adjuvant Chemoradiation Following Chemotherapy in Resected Pancreatic Cancer Patients With Lymph Node Metastasis or R1 Resection}

\author{
Jiazhang Xing ${ }^{1}$, Bo Yang ${ }^{2}$, Xiaorong Hou ${ }^{2}$, Ning Jia ${ }^{1}$, Xiaolei Gong ${ }^{1}$, Xiaoyuan $\mathrm{Li}^{1}$, \\ $\mathrm{Na}$ Zhou $^{1}$, Yuejuan Cheng ${ }^{1 * t}$ and Chunmei Bai ${ }^{1 \star t}$ \\ ${ }^{1}$ Department of Medical Oncology, Peking Union Medical College Hospital, Chinese Academy of Medical Sciences, \\ Beijing, China, 2 Department of Radiotherapy, Peking Union Medical College Hospital, Chinese Academy of Medical \\ Sciences, Beijing, China
}

Pancreatic ductal adenocarcinoma (PDAC) is a lethal disease with a poor prognosis. In resectable PDAC, the recurrence rate is still high even when surgery and adjuvant chemotherapy (CT) are applied. Regional lymph node metastasis and positive margins are associated with higher recurrence risk and worse survival. Adjuvant radiotherapy has been explored, but its efficacy remains controversial. In recent years, some characteristics have been reported to stratify patients who may benefit from adjuvant chemoradiation (CRT), such as lymph node metastasis and margin status. Adjuvant chemotherapy followed by chemoradiation (CT-CRT) was also proposed. A total of 266 patients with resectable PDAC who have lymph node metastasis or $\mathrm{R} 1$ resection after surgery were enrolled. In multivariate Cox regression analyses, pancreatic body or tail tumor location (HR 0.433, $\mathrm{p}<0.0001$, compared with pancreatic head) and adjuvant CT predicted a better survival, while there were no significant differences among the different CT regimens. Higher T stage indicated poor survival (stage I: reference; stage II: HR 2.178, $\mathrm{p}=0.014$; stage III: HR 3.581, $\mathrm{p}=0.001$ ). Propensity score matching was applied in 122 patients to explore the role of CRT. A cohort of 51 patients ( 31 and 20 patients in the CT and CT-CRT groups, respectively) was generated by matching. Further analyses revealed adjuvant CT-CRT was associated with prolonged survival compared with CT alone (HR $0.284, p=0.014$ ) and less frequent local recurrences ( $56.5 \%$ vs. $21.4 \%$ in the CT and CTCRT group, respectively). However, no significant differences in disease-free survival among these two groups were observed.

Keywords: adjuvant chemotherapy, adjuvant chemoradiation, pancreatic ductal adenocarcinoma, lymph node metastasis, $\mathbf{R} 1$ resection 


\section{INTRODUCTION}

Pancreatic ductal adenocarcinoma (PDAC) is highly malignant with a dismal prognosis. Although surgery is the only potentially curative method, up to $80 \%$ of patients who undergo curative resection experience recurrence within two years, and the 5-year survival rate is $10-25 \%(1,2)$. Lymph node metastasis and positive margins indicate a poor prognosis. Lymph node metastasis is associated with worse disease-free survival (DFS) and overall survival (OS) (3, 4). R1 resection is also associated with decreased OS (5). Adjuvant chemotherapy (CT) can improve long-term outcomes compared with observation. Based on the PRODIGE-24 and ESPAC-4 trials (6, 7), six months of mFOLFIRINOX or gemcitabine with capecitabine is the recommended adjuvant CT regimen. Gemcitabine monotherapy is an alternative option for patients who cannot tolerate combination therapy. In the Asian population, S-1 was shown to markedly prolong OS with lower toxicity than gemcitabine (8). However, after receiving surgical treatment and standard adjuvant CT, patients are still at high risk of local relapse (6). Therefore, adjuvant radiotherapy has been investigated for many years in the treatment of resectable pancreatic adenocarcinoma.

Due to the lack of high-quality clinical trials, the role of radiotherapy is still controversial. Adjuvant chemoradiation (CRT) alone did not show any OS benefit over observation due to the toxicity of radiotherapy and the delay of the administration of CT (9-11). Therefore, some researchers have investigated the role of adjuvant chemotherapy and chemoradiation (CT-CRT). However, for non-selected patients, no differences in DFS and OS were observed between adding CRT to adjuvant CT and administering CT alone according to several phase 2 and phase 3 trials $(12,13)$. Patients with certain pathological characteristics may benefit from additional CRT. Lymph node metastasis favors improved OS from CT-CRT when compared to CT or CRT alone ( 0 positive nodes: hazard ratio (HR) $0.96, \mathrm{P}=0.67$; $1-3$ positive nodes: HR 0.74, $\mathrm{P}<0.001$; $4+$ positive nodes: HR $0.75, \mathrm{P}<0.001$ ) (14). Positive resection margin is also an indicator of survival benefit from CRT (15). Therefore, the American Society of Clinical Oncology (ASCO) guidelines (16) recommend that adjuvant CRT may be offered to patients with $\mathrm{R} 1$ resection or node-positive disease after 4 to 6 months of systemic adjuvant CT. However, the view of the role of CT-CRT is not consistent among different international academic societies, and the European Society for Medical Oncology (ESMO) guidelines do not recommend adjuvant CT-CRT (17).

Our study enrolled 266 patients with R1 resection or nodepositive pancreatic cancer after surgery. Survival analysis based on baseline characteristics and treatment was performed. Propensity score matching (PSM) was used to investigate the role of radiotherapy in 122 patients who had medical records of adjuvant therapy.

\section{METHODS}

\section{Patients}

This study was reviewed and approved by the ethics committee of Peking Union Medical College Hospital (PUMCH). The study sample comprised consecutive patients who received a pathological diagnosis of pancreatic adenocarcinoma and underwent curative-intent surgery between 1 January 2015 and 1 October 2019 at PUMCH. All enrolled patients had lymph node metastasis or $\mathrm{R} 1$ resection. $\mathrm{R} 1$ resection was defined as a positive margin within less than $1 \mathrm{~mm}$ according to the $8^{\text {th }}$ American Joint Committee on Cancer (AJCC) manual (18).

The exclusion criteria were a history of other cancers, R2 resection and distant metastasis before surgery. Pathologic characteristics, including tumor location, tumor size, lymph node metastasis and margin status, were collected from the original pathology reports. Clinical characteristics, including sex, age at diagnosis, CA19-9 before CT, CT information, radiotherapy information and follow-up records, were obtained from detailed clinical records. All the patients were restaged pathologically according to the 8th AJCC TNM classification (18). The follow-up duration was from the surgery date to 1 October 2020.

Patients with CA19-9 levels lower than three times the upper limit of normal before CT and having adjuvant treatment records in the hospital were selected to perform further PSM analysis. The patients were categorized into two groups: the CT group and the CT-CRT group. The CT-CRT group only enrolled patients who did not have distant metastasis before radiotherapy. Firstrecurrence sites were analyzed in the after-matching cohort. By radiologic records, local recurrence was classified as recurrences in the remnant pancreas, the surgical bed, or locoregional nodes. Other recurrences outside these areas were defined as distant recurrence (19). The DFS was calculated as the time from surgery to the first event of recurrent disease, death, or last follow-up.

\section{Statistical Analysis}

Statistical analyses were performed using SPSS version 26.0 (IBM Corp., NY). Continuous variables are summarized as median values with interquartile range, and categorical outcome variables are summarized as frequencies with percentages. The Kaplan-Meier method was used to investigate survival in different treatment modalities, CT regimens, pathological $\mathrm{T}$ and $\mathrm{N}$ staging and TNM stage groups. The Cox proportional hazards method was used to select significant independent predictors for survival. Multiple linear regression was applied to analyze the correlation of variables in the Cox regression model. Only factors with a p-value less than 0.1 in the univariate Cox regression analyses were included in further multivariate Cox regression analyses.

The PSM method was used to balance the potential confounding factors between the CT and CT-CRT groups. A multivariable logistic regression model was used to create propensity scores that included the following covariates: age, sex, tumor location, margin status, pathological $\mathrm{T}$ and $\mathrm{N}$ staging, TNM stage, pre-CT CA19-9, CT regimen and cumulative dose. A caliper of 0.2 propensity score standard deviations was used. Patients in the adjuvant CT-CRT group were matched at a ratio of 1:2 to patients in the adjuvant CT group. Differences in patient characteristics among the matched groups were evaluated using Student's independent-sample T-test and the chi-square test. 


\section{RESULTS}

\section{Patients}

This study enrolled 266 patients with lymph node metastasis or R1 resection after pancreatic adenocarcinoma surgery. The median age at diagnosis was 61.5 years. A total of 148 (55.6\%) of the enrolled patients were male. A total of $69.2 \%$ of patients had tumors located in the pancreatic head. Ninety-one patients had an R1 resection. Most patients had stage T2 tumor and TNM stage II disease. Regarding lymph node status, few patients (7.5\%) did not have lymph node metastasis, while $63.9 \%$ of patients had N1 stage and $28.6 \%$ of patients had N2 stage. The main CT regimens used were gemcitabine monotherapy (GEM), S-1 monotherapy, and gemcitabine plus oral fluoropyrimidine combination regimen. S-1 was the most commonly used oral fluoropyrimidine drug in our study, while capecitabine was also used. Thirty-seven patients did not receive CT because of intolerance or unwillingness. A total of $9.4 \%$ of patients received adjuvant fluoropyrimidine-based CRT (radiation dose: median total dose: $50.0 \mathrm{~Gy}$, range: 45.0-56.0 Gy; median fraction dose: 1.8 Gy, range: 1.8-2.0 Gy) after 2-6 cycles of CT. The baseline characteristics are provided in Table 1. Among the 266 patients, 122 patients had adjuvant treatment records including regimen, dose and cycles in our center.

\section{OS Analysis}

Survival time of the enrolled patients ranged from 3 to 55 months, with a median of 21 months. OS rates at 1,3 , and 5 years of $77.1 \%$, $32.3 \%$, and $15.7 \%$, respectively. The median follow-up time was 18 months (range 3-85), with 218 (82.0\%) patients showing a response. The median OS for patients receiving CT-CRT was 52.0 months (95\% Cl: 38.5-65.5) compared with 20.0 months for those receiving CT $(95 \% \mathrm{Cl}$ : 17.6-22.4). The median OS for patients with tumors located in the pancreatic head was 19.0 months (95\% Cl: 15.8-22.2), while for patients with tumors located in the pancreatic body or tail, it was 29.0 months (95\% Cl: 15.842.2) (Figure 1A). The median OS times for patients who received GEM alone, gemcitabine with S-1 (GS) and S-1 monotherapy were 28.0 months (95\% Cl: 19.2-36.8), 25.0 months (95\% Cl: 16.2-33.8) and 23.0 months (95\% Cl: 12.1-33.9), respectively (Figure 1B). Four patients received mFOLFIRINOX therapy, three of whom died, and their survival times were 8.0, 14.0 and 17.0 months. The median OS for patients with stage T2 and T3 tumors was 20.0 months, with $95 \% \mathrm{Cl}$ values of 17.1-22.3 and 16.1-23.9, respectively. Patients with stage T1 tumors had better survival, with an OS of 37.0 months and a $50.8 \%$ cumulative survival rate (Figure 1C). According to the univariate Cox regression analyses, seven factors had a p-value less than 0.1 (Table 2). By multiple linear regression analysis, there was a correlation between the TNM stage and lymph node stage. Because the T stage and lymph node stage were included, the TNM stage was removed to modified our multivariable Cox regression model. Multivariate Cox regression analyses (Table 2) revealed that pancreatic body or tail tumor location (HR 0.434, $\mathrm{p}<0.0001$, compared with pancreatic head), adjuvant CT-CRT (HR 0.369, $\mathrm{p}=0.015$, compared with CT alone) and CT (GEM: HR 0.356, $\mathrm{p}<0.0001$; GS: HR 0.269, p<0.0001; S-1: HR 0.325, p<0.0001, compared with
TABLE 1 | Baseline characteristics of 266 patients with lymph node metastasis or $\mathrm{R} 1$ resection after surgery for resectable pancreatic adenocarcinoma.

\begin{tabular}{|c|c|c|}
\hline Characteristics & N (266) & $\%$ \\
\hline Age, years, median (IQR) & $61.5(55.0-67.0)$ & \\
\hline \multicolumn{3}{|l|}{ Sex } \\
\hline Male & 148 & 55.6 \\
\hline Female & 118 & 44.4 \\
\hline \multicolumn{3}{|l|}{ Location } \\
\hline Head & 184 & 69.2 \\
\hline Body/tail & 82 & 30.8 \\
\hline \multicolumn{3}{|l|}{ Margin status } \\
\hline RO & 175 & 65.8 \\
\hline $\mathrm{R} 1$ & 91 & 34.2 \\
\hline \multicolumn{3}{|l|}{ AJCC TNM stage } \\
\hline Stage I & 15 & 5.6 \\
\hline Stage ॥ & 175 & 65.8 \\
\hline Stage III & 76 & 28.6 \\
\hline \multicolumn{3}{|l|}{ AJCC T stage } \\
\hline $\mathrm{T} 1$ & 47 & 17.7 \\
\hline $\mathrm{T} 2$ & 167 & 62.8 \\
\hline T3 & 52 & 19.5 \\
\hline \multicolumn{3}{|l|}{ AJCC lymph node stage } \\
\hline NO & 20 & 7.5 \\
\hline N1 & 170 & 63.9 \\
\hline N2 & 76 & 28.6 \\
\hline \multicolumn{3}{|l|}{ Chemotherapy regimen } \\
\hline None & 37 & 13.9 \\
\hline GEM & 64 & 24.1 \\
\hline GS & 82 & 30.8 \\
\hline S-1 & 51 & 19.2 \\
\hline mFOLFIRINOX & 4 & 1.5 \\
\hline Unknown & 28 & 10.5 \\
\hline \multicolumn{3}{|l|}{ Radiotherapy } \\
\hline No & 241 & 90.6 \\
\hline Yes & 25 & 9.4 \\
\hline
\end{tabular}

GEM, gemcitabine monotherapy; GS, gemcitabine plus oral fluoropyrimidine combination regimen.

no CT) were significant independent predictors for better OS. Higher T stage indicated poor survival (stage I: reference; stage II: HR 2.199, $\mathrm{p}=0.013$; stage III: HR 3.796, $\mathrm{p}<0.0001)$.

\section{PSM}

A total of 122 patients with adjuvant treatment records after surgery were categorized into two groups: the CT group (97 patients) and the CT-CRT group (25 patients). The matching procedure was successfully improved balance in age, sex, tumor location, marginal status, AJCC TNM staging, pre-CT CA19-9, CT regimen and cumulative CT dose between the two groups. It yielded a cohort of 51 patients ( 31 and 20 patients in the CT group and CT-CRT group, respectively) for further analyses (Table 3). Because few patients in the CT-CRT group received S- 1 and sequential CRT, the CT regimens only included GEM and GS after matching.

Thirty-seven patients in the after-matching cohort experienced disease recurrence, 23 patients from the CT group $(74.2 \%)$ and 14 from the CT-CRT group (70\%). 56.5\% of recurrences in the CT group were local, $26.1 \%$ were distant, and $17.4 \%$ were both local and distant. In the CT-CRT group, $21.4 \%$ of recurrences were local, $50 \%$ were distant, and $28.6 \%$ were local and distant. Patients in the CTCRT group were less likely to have local recurrences than those in the CT group. Median DFS in the CT-CRT group was 19.0 months 


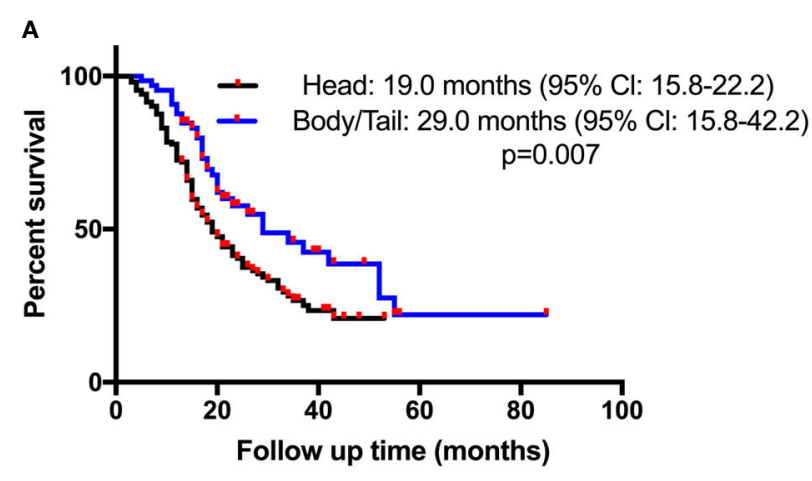

B

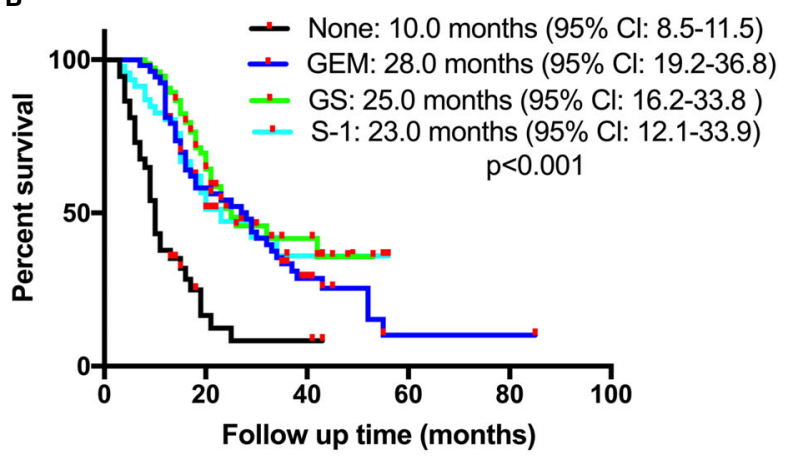

C

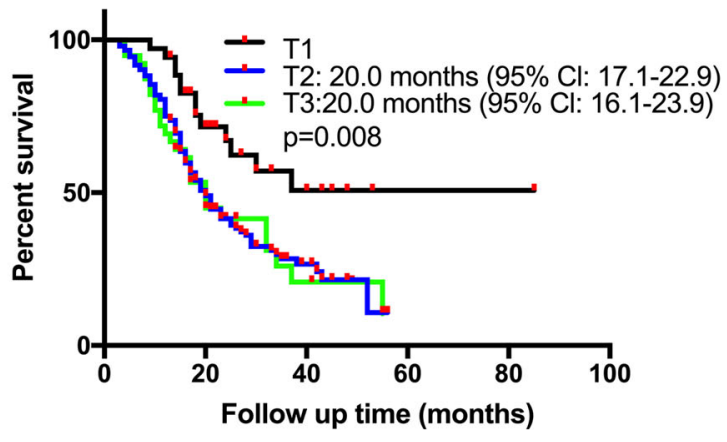

FIGURE 1 | Kaplan-Meier overall survival (OS) curves in patients with resectable pancreatic adenocarcinoma who underwent surgery and had lymph node metastasis or $\mathrm{R} 1$ resection based on (A) tumor location, (B) chemotherapy, or (C) pathological T stage. GEM, gemcitabine monotherapy; GS, gemcitabine plus oral fluoropyrimidine combination regimen

(95\% CI, 15.2 to 22.6 months) versus 14.0 months (95\% CI, 9.6 to 18.4 months) in the CT group, while no significant differences among these two groups were observed.

According to the univariate Cox regression analysis, the HR derived for the adjuvant CT-CRT cohort compared with the adjuvant CT alone cohort was $0.284(0.104-0.778)$, with a p-value of 0.014 , indicating that adjuvant CT-CRT was a significant prognostic risk factor for a more favorable OS. After matching, the median OS time for patients receiving
CT-CRT was 52.0 months (95\% Cl: 39.0-65.0) compared with 28.0 months for those receiving CT (95\% Cl: 16.3-40.0) (Figure 2). Because other factors in univariate Cox regression analysis did not show significance, further multivariate Cox regression analyses were not performed.

\section{DISCUSSION}

Patients with lymph node metastasis or R1 resection after surgery had a poor prognosis (3-5). These two factors were also reported as possible indicators for selecting patients who may benefit from adjuvant radiotherapy $(14,15)$. Therefore, in our study, we focused on these patients to analyze possible prognostic factors and explored the role of postoperative CT-CRT.

In our analyses, pancreatic tumors located in the pancreatic body or tail indicated a better prognosis than tumors located in the pancreatic head for patients undergoing curative resection. Several studies reported similar results (20-22). Pathologically unfavorable is a possible explanation for the worse OS associated with tumors located in the pancreatic head, which tend to have lymph node metastases, advanced pathologic stages and worse tumor grades (21). We also found that the pathological T stage was a significant prognostic factor. Indeed, the significance of tumor size in clinical outcomes has received more attention since the reversion of the $\mathrm{T}$ stage in the 8th edition of the AJCC TNM staging system (18). Many studies have validated the more detailed T staging's superiority in stratifying patients by survival $(23,24)$. Larger tumors correlated with higher CA19-9 levels, higher grades, perineural invasion, R1 resection and more positive lymph nodes (25). Our study indicated that tumor location and size should be given more attention in patients with $\mathrm{R} 1$ resection or positive lymph nodes. The role of tumor location and size in treatment stratification for these high recurrence risk patients could be further explored. In this study, the $\mathrm{N}$ and TNM stage were significantly associated with survival in univariate Cox regression analyses but not in multivariate analyses. The few N0 and TNM stage I patients led to the patients' uneven stage distribution, contributing to the bias. An association between marginal status (R0 vs R1) after resection and OS was not found. The criteria we set for patient selection resulted in all patients with $\mathrm{R} 0$ resection included in this study having lymph node metastasis, which also indicated a poor prognosis. Thus, the survival difference between different marginal status groups was not revealed. The CT regimen was also recorded and analyzed in our study. For patients after resection of pancreatic adenocarcinoma, mFOLFIRINOX or gemcitabine with capecitabine is the recommended adjuvant CT regimen, although the increased toxicities of mFOLFIRINOX limit its widespread use (7). Gemcitabine monotherapy is an alternative option. In the Asian population, the S-1 regimen was indicated to markedly prolong OS with lower toxicity than gemcitabine (8). In our study, only four patients received the mFOLFIRINOX regimen, and most of the patients included received the GEM, GS or S-1 regimen. CT showed a significant improvement in OS compared with observation, while there were no significant differences among the different CT regimens.

Due to the high risk of pancreatic cancer recurrence after surgery and CT (6), the effectiveness of adjuvant radiotherapy has been 
TABLE 2 | Cox proportional hazards regression analysis of the risk of death among 266 patients with lymph node metastasis or R1 resection after surgery for resectable pancreatic adenocarcinoma.

\begin{tabular}{|c|c|c|c|c|c|c|}
\hline \multirow[t]{2}{*}{ Characteristics } & \multicolumn{3}{|c|}{ Univariate analysis } & \multicolumn{3}{|c|}{ Multivariate anlaysis } \\
\hline & HR & $95 \% \mathrm{Cl}$ & $P$ value & HR & $95 \% \mathrm{Cl}$ & P value \\
\hline Age & 1.015 & $0.998-1.033$ & 0.081 & 1.020 & $1.000-1.040$ & 0.053 \\
\hline \multicolumn{7}{|l|}{ Sex } \\
\hline Male & Ref & & & & & \\
\hline Female & 0.919 & $0.652-1.294$ & 0.628 & & & \\
\hline \multicolumn{7}{|l|}{ Location } \\
\hline Head & Ref & & & Ref & & \\
\hline Body/tail & 0.584 & $0.392-0.871$ & 0.008 & 0.434 & $0.275-0.685$ & $<0.0001$ \\
\hline \multicolumn{7}{|l|}{ Margin status } \\
\hline RO & Ref & & & & & \\
\hline $\mathrm{R} 1$ & 1.000 & $0.696-1.436$ & 1.000 & & & \\
\hline \multicolumn{7}{|l|}{ AJCC TNM stage } \\
\hline Stage I & Ref & & & & & \\
\hline Stage II & 2.197 & $0.890-5.422$ & 0.088 & & & \\
\hline Stage III & 2.663 & $1.044-6.796$ & 0.040 & & & \\
\hline \multicolumn{7}{|l|}{ AJCC T stage } \\
\hline T1 & Ref & & & Ref & & \\
\hline $\mathrm{T} 2$ & 2.348 & $1.313-4.198$ & 0.004 & 2.199 & $1.183-4.086$ & 0.013 \\
\hline T3 & 2.440 & $1.257-4.733$ & 0.008 & 3.796 & $1.856-7.756$ & $<0.0001$ \\
\hline \multicolumn{7}{|c|}{ AJCC lymph node stage } \\
\hline NO & Ref & & & Ref & & \\
\hline N1 & 1.896 & $0.871-4.124$ & 0.107 & 1.513 & $0.682-3.358$ & 0.309 \\
\hline N2 & 2.293 & & & 1.748 & $0.753-4.060$ & 0.194 \\
\hline \multicolumn{7}{|c|}{ Chemotherapy regimen } \\
\hline None & Ref & & & & & \\
\hline GEM & 0.318 & $0.196-0.515$ & $<0.0001$ & 0.356 & $0.223-0.598$ & $<0.0001$ \\
\hline GS & 0.234 & $0.143-0.382$ & $<0.0001$ & 0.269 & $0.160-0.451$ & $<0.0001$ \\
\hline S-1 & 0.286 & $0.167-0.491$ & $<0.0001$ & 0.325 & $0.187-0.564$ & $<0.0001$ \\
\hline mFOLFIRINOX & 0.517 & 0.158-1.694 & 0.276 & 0.690 & $0.198-2.410$ & 0.561 \\
\hline \multicolumn{7}{|l|}{ Radiotherapy } \\
\hline No & Ref & & & Ref & & \\
\hline Yes & 0.286 & $0.132-0.618$ & 0.001 & 0.369 & $0.165-0.826$ & 0.015 \\
\hline
\end{tabular}

HR, hazard ratio; $\mathrm{Cl}$, confidence interval; Ref, reference.

GEM, gemcitabine monotherapy; GS, gemcitabine plus oral fluoropyrimidine combination regimen.

explored for many years. However, compared with CT, the role of radiotherapy in the adjuvant setting is controversial. In our study, PSM was applied and generated a cohort for further analysis (31 patients in the CT group and 20 patients in the CT-CRT group). According to the following Cox regression analysis, adjuvant CTCRT was a significant prognostic factor for a more favorable OS $(\mathrm{p}=0.014)$. Some studies also supported the survival benefit of CTCRT (26). The prolonged OS of the CT-CRT group might benefit from local control improvement. The local recurrence rate of the CTCRT group was lower than that of the CT group. The improvement of local control by adding CRT in pancreatic cancer therapy was illustrated in other studies. In a phase II prospective randomized study comparing adjuvant CT alone and adjuvant CT-CRT, the local and simultaneous local and distant progression rates were $11 \%$ and $13 \%$, respectively, in the CT-CRT group, which were lower than those (24\% and 20\%) in the CT group (12). Other retrospective studies also reached similar results (26-28). According to previous studies, the risk of local recurrence correlated with lymph node metastasis (19, 29) and R1 resection (30-32). The better outcome in the CT-CRT group in this study, of which patients have lymph node metastasis or $\mathrm{R} 1$ resection, might indicate the importance of patients selecting. Patients who have a higher risk of local recurrence were likely to benefit from CT-CRT. Meanwhile, some pathological characteristics have been reported to indicate benefit from adding adjuvant radiotherapy, such as pT3 (33), lymph node metastasis $(14,29,33$, 34) and positive margin status (35).

In the ESPAC 4 study, for patients with a positive margin, gemcitabine combined with capecitabine did not significantly improve patients' overall survival compared to gemcitabine alone (23.7 vs. 23.0 months) (6). Although the mFOLFIRINOX regimen showed higher activity in nodal positive or $\mathrm{R} 1$ resection pancreatic cancer, few patients could tolerate the toxicities. Therefore, adjuvant radiotherapy could be taken into consideration in these patients. Our study focused on these patients for the first time to analyze and prove the survival benefit of CT-CRT. According to previous studies, the delayed administration of CT, toxicities of radiotherapy (9-11) and radiation dose were potential reasons for the lack of OS benefit observed in CRT or CT-CRT (36). In our study, all patients received a standard regimen, including CRT following 4-6 months of CT, recommended by the ASCO guidelines (16). Intensity-modulated radiation therapy (IMRT) was used in all patients with a sufficient radiotherapy dose (median total dose of $50 \mathrm{~Gy}$ and fraction dose of 1.8 Gy). IMRT has been widely used in recent years to achieve more conformal dose delivery with less toxicity. In pancreatic cancer, 
TABLE 3 | Baseline characteristics before and after propensity score matching.

\begin{tabular}{|c|c|c|c|c|c|c|}
\hline \multirow[b]{2}{*}{ Characteristics } & \multicolumn{3}{|c|}{ Before PSM } & \multicolumn{3}{|c|}{ Aefore PSM } \\
\hline & CT (n = 97) & CT-CRT $(n=25)$ & $P$ value & CT $(n=31)$ & CT-CRT $(n=20)$ & $P$ value \\
\hline Age(mean \pm SD) & $60.7 \pm 9.8$ & $60.3 \pm 11.8$ & 0.87 & $57.5 \pm 10.8$ & $58.8 \pm 11.5$ & 0.69 \\
\hline Sex & & & 0.70 & & & 0.97 \\
\hline Male & 54 & 15 & & 20 & 13 & \\
\hline Female & 43 & 10 & & 11 & 7 & \\
\hline Location & & & 0.70 & & & 0.94 \\
\hline Head & 66 & 16 & & 22 & 14 & \\
\hline Body/tail & 31 & 9 & & 9 & 6 & \\
\hline Marginal status & & & 0.05 & & & 0.81 \\
\hline RO & 70 & 13 & & 16 & 11 & \\
\hline $\mathrm{R} 1$ & 27 & 2 & & 15 & 9 & \\
\hline AJCC TNM stage & & & 0.38 & & & 0.83 \\
\hline Stage I & 5 & 3 & & 2 & 2 & \\
\hline Stage ॥ & 73 & 16 & & 21 & 12 & \\
\hline Stage III & 19 & 6 & & 8 & 6 & \\
\hline \multicolumn{7}{|l|}{ AJCC T satge } \\
\hline T1 & 14 & 8 & 0.12 & 8 & 7 & 0.70 \\
\hline $\mathrm{T} 2$ & 62 & 13 & & 18 & 11 & \\
\hline T3 & 21 & 4 & & 5 & 2 & \\
\hline \multicolumn{7}{|l|}{ AJCC lymph node stage } \\
\hline NO & 6 & 4 & 0.21 & 2 & 3 & 0.52 \\
\hline N1 & 72 & 15 & & 21 & 11 & \\
\hline N2 & 19 & 6 & & 8 & 6 & \\
\hline \multicolumn{7}{|l|}{ Pre-chemotherapy CA199 } \\
\hline$>37$ & 30 & 7 & 0.78 & 9 & 5 & 0.75 \\
\hline$\leq 37$ & 67 & 18 & & 22 & 15 & \\
\hline \multicolumn{7}{|l|}{ Chemotherapy regimen } \\
\hline GEM & 36 & 11 & 0.39 & 16 & 10 & 0.91 \\
\hline GS & 37 & 11 & & 15 & 10 & \\
\hline S-1 & 24 & 3 & & 0 & 0 & \\
\hline \multicolumn{7}{|c|}{ Chemotherapy cumulative dose(mg) } \\
\hline Gemcitabine(Median, IQR) & $21300(14400)$ & 24900 (19200) & 0.13 & $22800(16800)$ & 25200 (19200) & 0.76 \\
\hline Fluropyrimidine(Median, IQR) & 8400 (3780) & $11760(9380)$ & 0.03 & 9120 (7840) & 10920 (9380) & 0.93 \\
\hline \multicolumn{7}{|l|}{ IMRT dose(Gy) } \\
\hline Median total dose & & 50 & & & 50 & \\
\hline Median fraction dose & & 1.8 & & & 2 & \\
\hline
\end{tabular}

GEM, gemcitabine monotherapy; GS, gemcitabine plus oral fluoropyrimidine combination regimen.

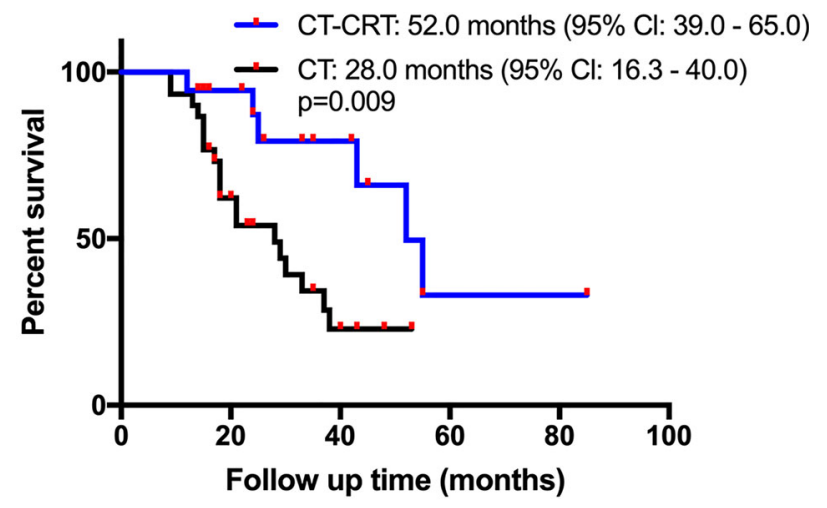

FIGURE 2 | Kaplan-Meier overall survival (OS) curves in patients with resectable pancreatic adenocarcinoma who underwent surgery and had lymph node metastasis or R1 resection after propensity score matching. Adjuvant chemotherapy (CT) vs adjuvant chemotherapy and chemoradiation (CT-CRT).
IMRT had significantly reduced toxicities without changes in the therapeutic outcome compared with conventional 3D-conformal radiation therapy (37). Thus, our study results could be more convincing by adapting reasonable regimens and advanced technology. For the CT regimen given before adjuvant CRT, there were no differences in DFS and OS between gemcitabine and fluoropyrimidine-based regimens $(38,39)$. However, a trend to extend OS in the gemcitabine group for the patients with pancreatic adenocarcinoma in the pancreatic head was reported (39). In our study, the number of patients who received GS was equal to those who received gemcitabine alone.

There are some limitations of our study. First, it was a retrospective study based on single center. Second, the small sample size of patients who received CT-CRT limited a definitive conclusion. Third, about half of the patients enrolled in our study received S1 chemotherapy, which markedly prolonged OS with low toxicity in Asian patients but not demonstrated in European and North American patients. Hence, our results should be cautiously illustrated when applied in non-Asian populations. 
Further studies are in need to illustrate the survival benefit in selected patients. Biomarkers such as genetic alteration, circulating tumor DNA or circulating tumor cells should also be incorporated to investigate the predictive biomarker for chemoradiation. Phase III trial aimed at offering more convincing evidence for CT-CRT is ongoing, in which patients receive adjuvant CRT [50.4 Gy in 28 fractions with concomitant 5-fluorouracil (5-FU)] after five cycles of adjuvant CT (40).

In conclusion, our study focused on a subgroup of patients with resected pancreatic cancer who had a worse prognosis due to lymph node metastasis or positive margin status. Among the baseline and pathological characteristics, tumor location and the $\mathrm{T}$ stage were significantly associated with prognosis. There was no significant difference in the effectiveness of different CT regimens. PSM and Cox regression analysis illustrated that adjuvant CT-CRT was associated with prolonged patient OS and lower local recurrence rate compared with CT alone.

\section{DATA AVAILABILITY STATEMENT}

The original contributions presented in the study are included in the article/supplementary material. Further inquiries can be directed to the corresponding authors.

\section{REFERENCES}

1. Fenocchio E, Filippi R, Lombardi P, Quara V, Milanesio M, Aimar G, et al. Is There a Standard Adjuvant Therapy for Resected Pancreatic Cancer? Cancers (Basel) (2019) 11(10):1547. doi: 10.3390/cancers11101547

2. Mizrahi JD, Surana R, Valle JW, Shroff RT. Pancreatic Cancer. Lancet (2020) 395(10242):2008-20. doi: 10.1016/s0140-6736(20)30974-0

3. Morales-Oyarvide V, Rubinson DA, Dunne RF, Kozak MM, Bui JL, Yuan C, et al. Lymph Node Metastases in Resected Pancreatic Ductal Adenocarcinoma: Predictors of Disease Recurrence and Survival. Br J Cancer (2017) 117 (12):1874-82. doi: 10.1038/bjc.2017.349

4. Lowder CY, Metkus J, Epstein J, Kozak GM, Lavu H, Yeo CJ, et al. Clinical Implications of Extensive Lymph Node Metastases for Resected Pancreatic Cancer. Ann Surg Oncol (2018) 25(13):4004-11. doi: 10.1245/s10434-0186763-4

5. Demir IE, Jager C, Schlitter AM, Konukiewitz B, Stecher L, Schorn S, et al. R0 Versus R1 Resection Matters After Pancreaticoduodenectomy, and Less After Distal or Total Pancreatectomy for Pancreatic Cancer. Ann Surg (2018) 268 (6):1058-68. doi: 10.1097/SLA.0000000000002345

6. Neoptolemos JP, Palmer DH, Ghaneh P, Psarelli EE, Valle JW, Halloran CM, et al. Comparison of Adjuvant Gemcitabine and Capecitabine With Gemcitabine Monotherapy in Patients With Resected Pancreatic Cancer (ESPAC-4): A Multicentre, Open-Label, Randomised, Phase 3 Trial. Lancet (2017) 389(10073):1011-24. doi: 10.1016/s0140-6736(16)32409-6

7. Conroy T, Hammel P, Hebbar M, Ben Abdelghani M, Wei AC, Raoul JL, et al. FOLFIRINOX or Gemcitabine as Adjuvant Therapy for Pancreatic Cancer. N Engl J Med (2018) 379(25):2395-406. doi: 10.1056/NEJMoa1809775

8. Uesaka K, Boku N, Fukutomi A, Okamura Y, Konishi M, Matsumoto I, et al. Adjuvant Chemotherapy of S-1 Versus Gemcitabine for Resected Pancreatic Cancer: A Phase 3, Open-Label, Randomised, Non-Inferiority Trial (JASPAC 01). Lancet (2016) 388(10041):248-57. doi: 10.1016/s0140-6736(16)30583-9

9. Smeenk HG, van Eijck CH, Hop WC, Erdmann J, Tran KC, Debois M, et al. Long-Term Survival and Metastatic Pattern of Pancreatic and Periampullary Cancer After Adjuvant Chemoradiation or Observation: Long-Term Results of EORTC Trial 40891. Ann Surg (2007) 246(5):734-40. doi: 10.1097/ SLA.0b013e318156eef3

\section{ETHICS STATEMENT}

The studies involving human participants were reviewed and approved by Peking Union Medical College Hospital. The patients/participants provided their written informed consent to participate in this study.

\section{AUTHOR CONTRIBUTIONS}

JX, YC and $\mathrm{CB}$ conceived and designed the study. BY and $\mathrm{XH}$ collected the clinical data. NJ, XG, XL and NZ finished patients' follow-up. JX and YC performed the statistical analyses. JX wrote the manuscript. YC and $\mathrm{CB}$ reviewed and revised the manuscript. All authors contributed to the article and approved the submitted version.

\section{FUNDING}

This study received grants from the Chinese Academy of Medical Sciences (CAMS) Initiative for Innovative Medicine (CAMSI2M) 2017-I2M-1-001.

10. Neoptolemos JP, Stocken DD, Friess H, Bassi C, Dunn JA, Hickey H, et al. A Randomized Trial of Chemoradiotherapy and Chemotherapy After Resection of Pancreatic Cancer. N Engl J Med (2004) 350(12):1200-10. doi: 10.1056/ NEJMoa032295

11. Neoptolemos JP, Dunn JA, Stocken DD, Almond J, Link K, Beger H, et al. Adjuvant Chemoradiotherapy and Chemotherapy in Resectable Pancreatic Cancer: A Randomised Controlled Trial. Lancet (2001) 358(9293):1576-85. doi: 10.1016/s0140-6736(01)06651-x

12. Van Laethem JL, Hammel P, Mornex F, Azria D, Van Tienhoven G, Vergauwe P, et al. Adjuvant Gemcitabine Alone Versus Gemcitabine-Based Chemoradiotherapy After Curative Resection for Pancreatic Cancer: A Randomized EORTC-40013-22012/FFCD-9203/GERCOR Phase II Study. J Clin Oncol (2010) 28(29):4450-6. doi: 10.1200/JCO.2010.30.3446

13. Schmidt J, Abel U, Debus J, Harig S, Hoffmann K, Herrmann T, et al. OpenLabel, Multicenter, Randomized Phase III Trial of Adjuvant Chemoradiation Plus Interferon Alfa-2b Versus Fluorouracil and Folinic Acid for Patients With Resected Pancreatic Adenocarcinoma. J Clin Oncol (2012) 30(33):407783. doi: $10.1200 /$ jco.2011.38.2960

14. Ma SJ, Hermann GM, Prezzano KM, Serra LM, Iovoli AJ, Singh AK. Adjuvant Chemotherapy Followed by Concurrent Chemoradiation Is Associated With Improved Survival for Resected Stage I-II Pancreatic Cancer. Cancer Med (2019) 8(3):939-52. doi: 10.1002/cam4.1967

15. Stocken DD, Buchler MW, Dervenis C, Bassi C, Jeekel H, Klinkenbijl JH, et al. Meta-Analysis of Randomised Adjuvant Therapy Trials for Pancreatic Cancer. Br J Cancer (2005) 92(8):1372-81. doi: 10.1038/sj.bjc.6602513

16. Khorana AA, Mangu PB, Berlin J, Engebretson A, Hong TS, Maitra A, et al. Potentially Curable Pancreatic Cancer: American Society of Clinical Oncology Clinical Practice Guideline. J Clin Oncol (2016) 34(21):2541-56. doi: 10.1200/ jco.2016.67.5553

17. Ducreux M, Cuhna AS, Caramella C, Hollebecque A, Burtin P, Goéré D, et al. Cancer of the Pancreas: ESMO Clinical Practice Guidelines for Diagnosis, Treatment and Follow-Up. Ann Oncol (2015) 26(Suppl 5):v56-68. doi: 10.1093/annonc/mdv295

18. Amin MB ES, Greene F. AJCC Cancer Staging Manual. 8th ed. New York: Springer (2016).

19. Jones RP, Psarelli EE, Jackson R, Ghaneh P, Halloran CM, Palmer DH, et al. Patterns of Recurrence After Resection of Pancreatic Ductal Adenocarcinoma: 
A Secondary Analysis of the ESPAC-4 Randomized Adjuvant Chemotherapy Trial. JAMA Surg (2019) 154(11):1038-48. doi: 10.1001/jamasurg.2019.3337

20. Meng Z, Cao M, Zhang Y, Liu Z, Wu S, Wu H. Tumor Location as an Indicator of Survival in T1 Resectable Pancreatic Ductal Adenocarcinoma: A Propensity Score-Matched Analysis. BMC Gastroenterol (2019) 19(1):59. doi: 10.1186/s12876-019-0975-3

21. Winer LK, Dhar VK, Wima K, Morris MC, Lee TC, Shah SA, et al. The Impact of Tumor Location on Resection and Survival for Pancreatic Ductal Adenocarcinoma. J Surg Res (2019) 239:60-6. doi: 10.1016/j.jss.2019.01.061

22. Song Y, Chen Z, Chen L, He C, Huang X, Duan F, et al. A Refined Staging Model for Resectable Pancreatic Ductal Adenocarcinoma Incorporating Examined Lymph Nodes, Location of Tumor and Positive Lymph Nodes Ratio. J Cancer (2018) 9(19):3507-14. doi: 10.7150/jca.26187

23. Allen PJ, Kuk D, Castillo CF, Basturk O, Wolfgang CL, Cameron JL, et al. MultiInstitutional Validation Study of the American Joint Commission on Cancer (8th Edition) Changes for T and N Staging in Patients With Pancreatic Adenocarcinoma. Ann Surg (2017) 265(1):185-91. doi: 10.1097/sla.0000000000001763

24. Schlitter AM, Jesinghaus M, Jäger C, Konukiewitz B, Muckenhuber A, Demir IE, et al. pT But Not pN Stage of the 8th TNM Classification Significantly Improves Prognostication in Pancreatic Ductal Adenocarcinoma. Eur J Cancer (2017) 84:121-9. doi: 10.1016/j.ejca.2017.06.034

25. Marchegiani G, Andrianello S, Malleo G, De Gregorio L, Scarpa A, MinoKenudson M, et al. Does Size Matter in Pancreatic Cancer?: Reappraisal of Tumour Dimension as a Predictor of Outcome Beyond the TNM. Ann Surg (2017) 266(1):142-8. doi: 10.1097/SLA.0000000000001837

26. Khawaja MR, Kleyman S, Yu Z, Howard T, Burns M, Nakeeb A, et al. Adjuvant Gemcitabine and Gemcitabine-Based Chemoradiotherapy Versus Gemcitabine Alone After Pancreatic Cancer Resection: The Indiana University Experience. Am J Clin Oncol (2017) 40(1):42-6. doi: 10.1097/ COC. 0000000000000115

27. Sainato A, Montrone S, Pasqualetti F, Coppola M, Cernusco NLV, Panichi M, et al. Adjuvant Chemoradiotherapy (Gemcitabine-Based) in Pancreatic Adenocarcinoma: The Pisa University Experience. Tumori (2017) 103 (6):577-82. doi: 10.5301/tt.5000664

28. Parikh AA, Maiga A, Bentrem D, Squires MH3rd, Kooby DA, Maithel SK, et al. Adjuvant Therapy in Pancreas Cancer: Does It Influence Patterns of Recurrence? J Am Coll Surg (2016) 222(4):448-56. doi: 10.1016/j.jamcollsurg.2015.12.031

29. Kamarajah SK, Sonnenday CJ, Cho CS, Frankel TL, Bednar F, Lawrence TS, et al. Association of Adjuvant Radiotherapy With Survival After MarginNegative Resection of Pancreatic Ductal Adenocarcinoma: A PropensityMatched National Cancer Database (NCDB) Analysis. Ann Surg (2019) 273 (3):587-94. doi: 10.1097/SLA.0000000000003242

30. Gnerlich JL, Luka SR, Deshpande AD, Dubray BJ, Weir JS, Carpenter DH, et al. Microscopic Margins and Patterns of Treatment Failure in Resected Pancreatic Adenocarcinoma. Arch Surg (2012) 147(8):753-60. doi: 10.1001/ archsurg.2012.1126

31. Groot VP, Rezaee N, Wu W, Cameron JL, Fishman EK, Hruban RH, et al. Patterns, Timing, and Predictors of Recurrence Following Pancreatectomy for Pancreatic Ductal Adenocarcinoma. Ann Surg (2018) 267(5):936-45. doi: 10.1097/SLA.0000000000002234

32. Honselmann KC, Pergolini I, Castillo FD, Deshpande V, Ting D, Taylor MS, et al. Timing But Not Patterns of Recurrence Is Different Between NodeNegative and Node-Positive Resected Pancreatic Cancer. Ann Surg (2020) 272:357-65. doi: 10.1097/SLA.0000000000003123
33. Rutter CE, Park HS, Corso CD, Lester-Coll NH, Mancini BR, Yeboa DN, et al. Addition of Radiotherapy to Adjuvant Chemotherapy Is Associated With Improved Overall Survival in Resected Pancreatic Adenocarcinoma: An Analysis of the National Cancer Data Base. Cancer (2015) 121(23):4141-9. doi: $10.1002 /$ cncr.29652

34. Suss NR, Talamonti MS, Bryan DS, Wang CH, Kuchta KM, Stocker SJ, et al. Does Adjuvant Radiation Provide Any Survival Benefit After an R1 Resections for Pancreatic Cancer? Surgery (2018) 163(5):1047-52. doi: 10.1016/ j.surg.2017.09.022

35. Osipov A, Nissen N, Rutgers J, Dhall D, Naziri J, Chopra S, et al. Redefining the Positive Margin in Pancreatic Cancer: Impact on Patterns of Failure, Long-Term Survival and Adjuvant Therapy. Ann Surg Oncol (2017) 24 (12):3674-82. doi: 10.1245/s10434-017-6076-z

36. Morganti AG, Falconi M, van Stiphout RG, Mattiucci GC, Alfieri S, Calvo FA, et al. Multi-Institutional Pooled Analysis on Adjuvant Chemoradiation in Pancreatic Cancer. Int J Radiat Oncol Biol Phys (2014) 90(4):911-7. doi: 10.1016/j.ijrobp.2014.07.024

37. Bittner MI, Grosu AL, Brunner TB. Comparison of Toxicity After IMRT and 3D-Conformal Radiotherapy for Patients With Pancreatic Cancer - a Systematic Review. Radiother Oncol (2015) 114(1):117-21. doi: 10.1016/ j.radonc.2014.11.043

38. Hsieh M-C, Chang W-W, Yu H-H, Lu C-Y, Chang C-L, Chow J-M, et al. Adjuvant Radiotherapy and Chemotherapy Improve Survival in Patients With Pancreatic Adenocarcinoma Receiving Surgery: Adjuvant Chemotherapy Alone Is Insufficient in the Era of Intensity Modulation Radiation Therapy. Cancer Med (2018) 7(6):2328-38. doi: 10.1002/cam4.1479

39. Regine WF, Winter KA, Abrams R, Safran H, Hoffman JP, Konski A, et al. Fluorouracil-Based Chemoradiation With Either Gemcitabine or Fluorouracil Chemotherapy After Resection of Pancreatic Adenocarcinoma: 5-Year Analysis of the U.S. Intergroup/RTOG 9704 Phase III Trial. Ann Surg Oncol (2011) 18(5):1319-26. doi: 10.1245/s10434-011-1630-6

40. Gemcitabine Hydrochloride With or Without Erlotinib Hydrochloride Followed by the Same Chemotherapy Regimen With or Without Radiation Therapy and Capecitabine or Fluorouracil in Treating Patients With Pancreatic Cancer That has Been Removed by Surgery. Available at: https://clinicaltrials.gov/ct2/show/ NCT01013649.

Conflict of Interest: The authors declare that the research was conducted in the absence of any commercial or financial relationships that could be construed as a potential conflict of interest.

Publisher's Note: All claims expressed in this article are solely those of the authors and do not necessarily represent those of their affiliated organizations, or those of the publisher, the editors and the reviewers. Any product that may be evaluated in this article, or claim that may be made by its manufacturer, is not guaranteed or endorsed by the publisher.

Copyright (c) 2021 Xing, Yang, Hou, Jia, Gong, Li, Zhou, Cheng and Bai. This is an open-access article distributed under the terms of the Creative Commons Attribution License (CC BY). The use, distribution or reproduction in other forums is permitted, provided the original author(s) and the copyright owner(s) are credited and that the original publication in this journal is cited, in accordance with accepted academic practice. No use, distribution or reproduction is permitted which does not comply with these terms. 